\begin{abstract}
NANODOSIMETRY AND NANODOSIMETRIC-BASED MODELS OF RADIATION
ACTION FOR RADON ALPHA PARTICLES
\end{abstract}

DR. MARCO ZAIDER

TECHNICAL REPORT

PROGRESS REPORT (JulY, 1990 - June, 1992)

1. A comprehensive biophysical theory applicable to radon

\title{
a particles
}

In the following we report on a theory for describing the biological effects of ionizing radiation, in particular radon $\alpha$ particles. Behind this approach is the recognition that biological effect; such as chromosome aberrations, cellular transformation, cellular inactivation, etc, are the result of a hierarchic sequence of radiation effects. We indicate how to treat each of the individual processes in this sequence, and also how to relate one effect to the hierarchically superior one.

\section{MASTER}




\section{A. Background}

The basic expression of this theory is a generalization of an equation originally proposed by kellerer and Rossi (1978). We write:

$$
\epsilon(D)=\epsilon_{s}(D) \int \frac{\left.t_{s}(x ; D) s(x) g^{\prime}, x\right)}{4 \pi x^{2}} d x \text {. }
$$

In this equation s stands for sublesion; it will be identified below with concrete molecular and genomic alterations. $t_{s}(x ; D) d x$ is the proximity function of sublesions and, by definition, is proportional to the probability that two sublesions are a distance $x$ apart. $s(x) d x$ is the proximity function of the sensitive matrix (i.e. cellular structures involved in radiation effects): it represents the geometry of genetic material. For instance, if one is interested in nucleotide damage, $s(x) d s$ is proportional to the distribution of distances between any pair of nucleotides in the DNA molecule. $g(x)$ is the probability that two sublesions at distance $x$ apart interact to produce a lesion. Again, if sublesions are ssb-s then $g(x)$ is the probability that two ssb-s separated by $x$ base pairs result in a dsb. Finding a numerical expression for $g(x)$ is one of the major goals of the theory.

$\epsilon(D)$ and $\epsilon_{s}(D)$ are, respectively, the number of lesions and sublesions at dose $D$. The general character of Eq(1) resides in the fact that no assumption is made concerning the mechanism by which sublesions are produced. The theory of dual radiation action (Kellerer and Rossi, 1978) is a particular case of Eq(1), namely when the yield of sublesions is proportional to the specific energy, $z$, and charged particles intersect the cellular volume randomly and independently. Then one obtains the linear-quadratic 
equation:

$$
\epsilon(D)=\alpha D+\beta D^{2}
$$

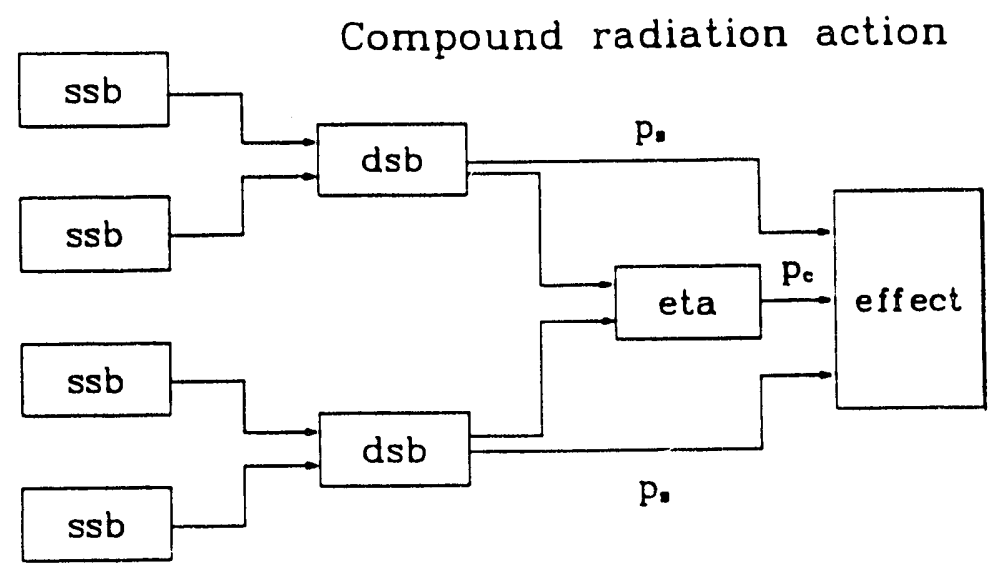

Figure 1 Schematic representation of the CRA concept: ssi-s interact to produce dsb-s; pairs of (unrepaired) dsb-s interact to produce exchange type chromosomal aberrations. Each "simple" process is described by Eq(1).

We have recently introduced the concept of compound radiation action, CRA (Rossi and zaider, 1992): It describes a sequence of two or more processes (in the sense that one process is the causative agent of the next one) each governed by an equation of the type, Eq(I). Fig.l illustrates schematically these concepts. The application of this theory to radon a particles (see below) is a good illustration for the need of an expression such as Eq(1) rather than the more conventional (and simpler) linear-quadratic formula, Eq(2). At the LET values typical for radon a particles saturation phenomena occur and, as indicated below, they preclude assuming proportionality between sublesion (ssb) production and energy locally deposited.

1B. From single- to double-strand breaks in DNA. Typical data for the production of DNA ssb and dsb are shown in 


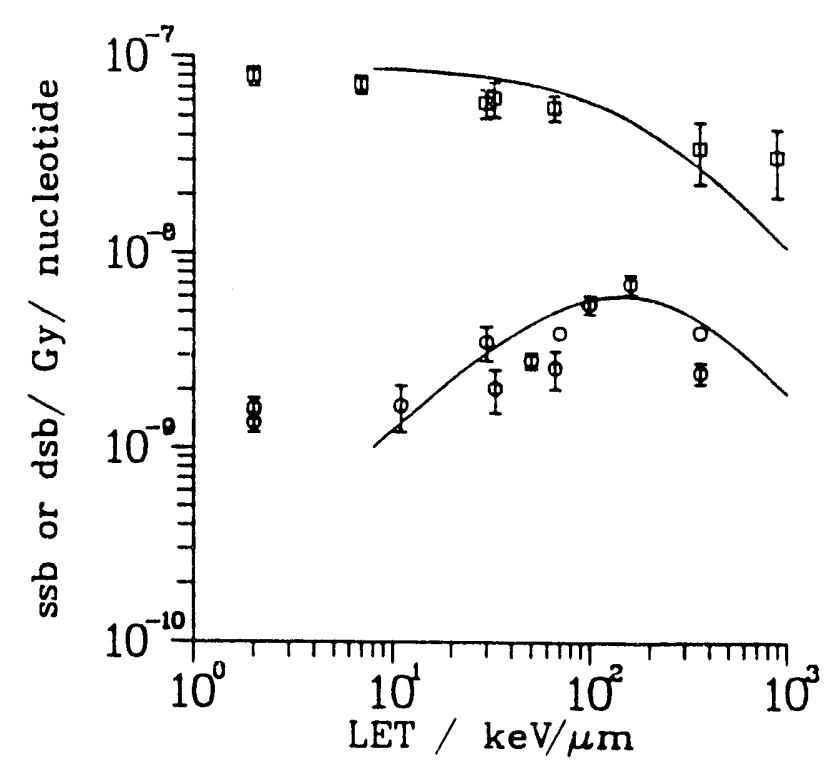

Figure 2 Left: Data for ssb (squares) and dsb (circles) from Kampf (1988). The solid curves are explained in the text.

Fig.2. This figure shows the low-dose limit of the number of breaks per unit dose and per nucleotide in v79/4 Chinese hamster cells (Rampf, 1988) as a function of LET. From the shape of the ssb curve (flat at low LET and descending at higher LET values) it appears that this process saturates as the local concentration of energy increases. We introduced a model (zaider, 1992) for the production of ssb-s and attempt to predict the shape of the dsb vs LET curve based on the ssb yield and the mechanism, Eq(1).

Concerning the ssb production it is assumed that the sensitive matrix, $M$, contains $N$ non-overlapping sites, $V_{a}$, which may contain each at most one ssb, and where the probability to produce a ssb is given by:

$$
p_{s a b}(r)=1-\exp \left[-k z_{a}(r)\right] \text {. }
$$

Here $k$ is a constant and $z_{a}(r)$ is the specific energy in a sphere of radius "a" centered at $r$. Neither the assumption of a spherical site nor the functional form of this equation are essential for what follows; they have been selected for simplicity only. We tentatively identify the sites with DNA nucieotides. 
The mean number of DNA ssb-s, $\epsilon_{\mathrm{ssb}}(D)$, in a cell exposed to a dose D is:

$$
\epsilon_{s s b}(D)=N \int\left[1-e^{-k z}\right] f_{a}(z ; D) d z
$$

In $E q(4) f_{a}(z ; D) d z$ is the multi-event microdosimetric spectrum in $V_{a}$. At the limit of $k z<<1$ we have $\epsilon_{s b b}(D)=k N D$, as expected. A more practical version of $\mathrm{Eq}(4)$ is (zaider, 1990):

$$
\epsilon_{s s b}(D)=N\left\{1-\exp \left[-\frac{D}{z_{1 F}} \int_{0}^{\infty}\left(1-e^{-k z}, f_{1}(z) d z\right]\right\}\right.
$$

where $z_{1 F}$ is the frequency mean of the single event spectrum $f_{1}(z) d z$. $p_{s s b}(r)$ of $E q(4)$ may be interpreted as a field of virtual ssb-s in the following sense: an ssb obtains at $r$ if this point coincides with the center of the site, $v_{a}$. similarly, one may define the field which consists of the centers of the sites, $v_{a}$ :

$$
I_{M}(r)=\sum \delta\left(r-r_{j}\right), \quad j=1,2, \ldots, N
$$

With each field one may associate a proximity function (kellerer, 1990). Thus, the proximity function of $I_{M}$ is:

$$
t_{M}(r)=N^{-1} \sum \delta\left(r-r_{j l}\right), \quad j, l=1,2, \ldots, N \quad j \neq l
$$

where $r_{j l}=\left|r_{j}-r_{1}\right|$. The proximity function of the field $p_{a b}(r)$, averaged over all directions of $r$ is (Kellerer, 1990):

$$
t_{s s b}(r) d r=I^{2} d r \int d \Omega_{r} \int d r^{\prime} p_{s s b}\left(I^{\prime}\right) p_{s s b}\left(r+I^{\prime}\right)
$$

Arguments similar to those used by Kellerer and Rossi (1978) lead to the following expression for the average yield of dsb-s: 


$$
\epsilon_{d s b}(D)=\epsilon_{s s b}(D) \int t_{M}(r) \frac{t_{s s b}(r ; D)}{4 \pi r^{2}} g(r) d r .
$$

$g(r)$ is the distance-dependent probability that two ssb-s yield a dsb. This is the basic result, Eq(1), corrected for saturation and applied to dsb production. Again, $t_{M}(r)$ is the proximity function for the nucleotides. There is a fundamental difference between $E q(9)$ and $E q(1)$ : in the former, the proximity function, $t_{s}$, includes the saturation effects.

To gain further insight in this formalism we apply now the results, Eqs $(5,9)$, to track segments (zaider, 1992). These are charged particles with negligible radial extension. Although for heavy ions this approximation may not be very good, many of the qualitative, and indeed quantitative, features of the formalism are retained, as will become apparent below. The mathematical results are given without demonstration.

The yield of DNA ssb-s at dose $D$ is given by:

$$
\epsilon_{s s b}(D)=D\left(\frac{3}{2} k N\right) \frac{1}{\alpha L}\left\{1+2 e^{-\alpha L}\left[\frac{1}{(\alpha L)^{2}}+\frac{1}{\alpha L}\right]-\frac{2}{(\alpha L)^{2}}\right\} .
$$

Here $L=L E T$ and $\alpha$ is a constant. The proximity function, $t,(r)$, may be obtained by numerical integration of an analytic expression, for details see zaider (1992). We take a spherical matrix, M, of radius $\Delta$ for which:

$$
t_{M}(r)=\frac{N}{\frac{4}{3} \pi \Delta^{3}} 4 \pi I^{2}\left(1-\frac{3 r}{4 \Delta}+\frac{r^{3}}{16 \Delta^{3}}\right)
$$

(11) and assume that two ssb-s within 
about 10 base pairs interact with probability p to produce a dsb. In this approximation both $\epsilon_{s s b}$ and $\epsilon_{d s b}$ depend on the product $\alpha L$ only. Calculated curves are shown in Fig.2. It is evident that, as predicted, the shape of the dsb curve can be accounted for in terms of ssb production only, without the need to invoke additional mechanisms.

1C. From DNA dsb to exchange-type chromosome aberrations (ETA). The analysis below is limited to data at $L E \mathrm{~T}<160 \mathrm{keV} / \mu \mathrm{m}$. We assume that in terms of dsb production the sensitive matrix may be divided in $N_{0}$ loci (volume $v$ each) with the property that only ssb$s$ in the locus contribute to the dsb yield (per locus). v should not be confused with the ssb production sites, $V_{a}$. A model of this type has been in fact suggested by Chatterjee and Holley (1991) who proposed that in a cell - as opposed to naked DNA - the internucleosomal region (i.e. DNA linker) is the main source of observable chromatin breaks (unrepaired dsb-s). It may be noted that, because there are typically 50-100 dsb/Gy per cell (ward, 1990), at radiobiologically relevant doses ( 1 to $10 \mathrm{~Gy}$ ) the probability of more than one dsb per locus is negligible. In what follows, the fact that in the dose range mentioned dsb-s result from single microdosimetric events only, is essential.

Let $z_{1}$ be the specific energy in $v$. Under the conditions described, the probability of a dsb in $v$ is:

$$
\operatorname{pdab}_{d}\left(z_{1}\right)=c z_{1}^{2} \text {, }
$$

where $c$ is a constant. On average:

$$
\mathrm{p}_{d s b}(D)=C z_{1 D} D \text {, }
$$

where $z_{10}$ is the dose-averaged single-event specific energy in $v$ and (in line with neglecting inter-track dsb production) the quadratic 
term in dose was omitted.

Exchange-type chromosome aberrations (ETA) obtain from the pairwise combination of chromatin breaks. Because dsb result from statistically independent events we can apply Eq(1) to calculate the yield, $\epsilon_{\text {ETA }}$ :

$$
\epsilon_{E T A}(D)=C \beta\left[D \int_{0}^{\infty} t_{d S b}(I) \gamma_{E T A}(I) d I+D^{2}\right]
$$

where we have absorbed all the constants in $C$ and

$$
\beta=\left(z_{10}\right)^{2}
$$

The expression, Eq(14), is a typical result of CRA in the sense that $\beta$ depends on radiation quality. This has been observed experimentally (Roots et al, 1985; Chapman et al, 1979). $\gamma_{\text {exa }}(r)$ is the probability that two dsb-s at distance $r$ apart interact to produce an ETA. $t_{d s b}(r) d r$ is the proximity function of chromosomal breaks.

A more general formulation of $\mathrm{Eq}(16)$ is:

$$
\sqrt{\beta}=\int_{0}^{\infty} t(r) \gamma_{d s b}(r) d r
$$

where $t(r)$ is the proximity function of energy deposition and $r_{d e b}$ is the probability that two energy transfers result in a dsb.

A very important consequence of Eq(14) is that, at low doses, the $\mathrm{RBE}$ of radiation 2 with respect to radiation 1 (proximity functions $t_{1}(r)$ and $t_{2}(r)$, respectively) must satisfy: 


$$
R B E_{E T A}=R B E_{D S B}^{2} \int t_{2}(I) \gamma_{E T A}(r) d r / \int t_{1}(r) \gamma_{E T A}(r) d r
$$

To the extent that $r(r)$ is a monotonically decreasing function of $r$, the REE for high-LET radiation must satisfy:

$$
R B E_{E T A}>R B E^{2} D S B
$$

CRA thus predicts a hierarchy of radiation effects.

To further evaluate the implications of the CRA formalism to the particular case of radon $\alpha$ particles we have use data for ssb/dsb production (Kampf, 1988) - see Fig.3 - together with results on ETA chromosome aberrations obtained on the same system $(\mathrm{V} 79 / 4)$ by Tolkendorf and Eichhorn (1983). We have re-analyzed the chromosome breakage data with the model, $E q(2)$, and obtained $\alpha$ and $\beta$ for 180 kVp $x$ rays and alpha particles (LET $=50,100,160 \mathrm{keV} / \mu \mathrm{m}$ ). In Fig.3 we compare the RBE for dsb induction (as reported in Kampf, 1988) with the RBE estimate from the $\checkmark \beta$ values for exchange-type aberrations (our analysis). Given the uncertainty in the determination of $\beta$ (the experimental points have been read off the published figures) the overall agreement between parameters for the two end points is good, and in line with the result, Eqs $(15,16)$. Also, the low-dose limit of the dicentric RBE verifies Eq(18). The function $\gamma_{\text {ETA }}(r)$ could not be obtained from the data.

The solid curves in Fig. 3 were calculated with the aid of Eq(16). For the radon alpha-particle proximity function we have used our Monte Carlo transport code (zaider et al, 1983). For the low-LET radiation we have used a proximity function derived by 


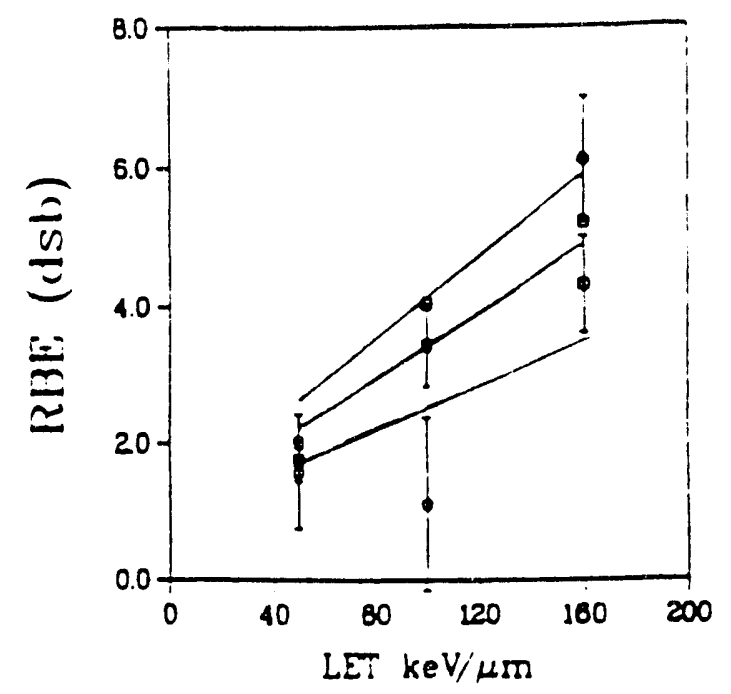

Figure 3 RBE for dsb production; data (squares) from Rampf (1988) and Tolkendorf and Eichorn (1983). The circles represent $\checkmark \beta$ for exchange-type aberrations (see text).

zaider et al (1982), namely $t_{x}(r)=1.54 \mathrm{r}^{-0.4}$, where the constants reflect the choice of units: $\mu \mathrm{m}$ for $r$ and $k e v / \mu \mathrm{m}$ for $t(r)$. We assumed a spherical volume, $v$, of diameter $d=60 \mathrm{~nm}$. Finally, the interaction probability of ssb-s was approximated with a Gausian:

$$
g(r)=\exp \left[-r^{2} / \sigma^{2}\right] \text {. }
$$

The curves in Fig. 3 were calculated at $y=2,3$ and $4 \mathrm{~nm}$, respectively and it appears that an interaction function with $\sigma=3 \mathrm{~nm}$ may account reasonably well for the data. This value is equivalent to some 15 base pairs, a result which agrees with the distance over which ssbs are known to interact in order to produce a dsb.

1D. Cellular inactivation

The correlation between cellular inactivation and chromosome breaks is well established (Barendsen, 1979). Although the exact contribution of each type of aberration to lethality is not known, both simple breaks and ETA are believed to be involved, the former by as much as $20 \%$ (C. Geard, personal communication). From what has 
been presented above, one may express the survival probability of a cell population as follows:

$$
-\log S(D)=\left(\lambda_{d B b} \alpha_{d s b}+\lambda_{E T A} \alpha_{E T A}\right) D+\lambda_{E T A} \beta D^{2} \text {. }
$$

The parameters $\lambda_{d s b}, \lambda_{x T A}$ are radiation independent. They represent, respectively, the fraction of dsb-s and ETA-s which contribute to lethality. $\alpha_{d s b}$ and $\alpha_{x \rightarrow A}$ indicate the low-dose limit of the dsb and ETA yields per unit dose; and $\beta_{\text {ETA }}$ is related to the dsb incidence according to $\mathrm{Eq}(16)$.

1E. A note on cellular transformation

It is improbable that oncological cell transformation is due to point mutations, and deletions (resulting in the elimination of suppressor genes) are a much more likely cause (Rossi, 1991). This implies that $\lambda_{d s b}$ is much less than $\lambda_{\text {ETA }}$ and that $R B E_{M}$ for transformation should be high. This would account for the fact that the $\mathrm{RBE}_{M}$ for cell killing is less than that for carcinogenesis which seems generally to be the case (ICRU, 1986).

\section{Charged particle transport and nanodosimetry of a particles}

2A. Electron transport in condensed water

The study of electron transport in condensed water is relevant for the radon proposed work for two reasons: a) $\alpha$-particle transport by Monte Carlo techniques must include transport of the secondary electrons ( $\delta$ rays), and $b$ ) many of the simulation techniques used in a particle transport are similar to those employed in the simulation of electron tracks.

Theoretical expressions for the interaction cross sections of charged particle with condensed media are presented in more detail 
in the first section of the Research Plan. The main result (zaider, 1991) is the link between the double-differential cross section for electron scattering resulting in energy loss $\omega$ and momentum transfer $q$, and the dielectric response function of the medium, $\epsilon(q, \omega):$

$$
\frac{d^{2} \sigma}{d \omega d q}=\frac{2}{\pi N v^{2}} \frac{1}{q} \operatorname{Im}\left[-\frac{1}{\epsilon(q, \omega)}\right]
$$

This expression is written in atomic units, $N$ is the total electron density in the scattering medium and $v$ is the projectile velocity. This equation shows clearly that in order to solve the charged particle transport problem one needs to evaluate $\epsilon(q, \omega)$.

Experimental information on $\epsilon(q, \omega)$ for biomaterials (including water) is rather limited; it is typically restricted to the $q=0$ region of the $(q, w)$ plane, as obtained from measurements of the refraction index or electron scattering (scattschneider, 1986). since electron transport requires cross sections at non-zero momentum transfer, it is necessary to evaluate $\epsilon(q, \omega)$ theoretically. The basis for this evaluation is (Raether, 1980):

$$
\epsilon_{x \alpha^{\prime}}(q, \omega)=\delta_{K K^{\prime}}+\frac{4 \pi e^{2}}{|q+K| i q^{\prime} \mid} \sum_{k, n, m} \frac{F_{(q+K) n m}(k) F_{m}^{*}}{\left.E_{m}(k+q)-E_{n}(k)-\omega-i \delta^{\prime}\right)}
$$

In this expression the crystal is described in terms of oneelectron wave functions $|n k\rangle$ of band index $n$, crystal wave vector $k$ and energy $E_{n}(k)$. $K$ and $K^{\prime}$ are reciprocal lattice vectors, $\&$ is the crystal volume and $\delta^{+}$is an infinitesimal positive number (the limit $\delta^{+} \rightarrow 0$ is taken at the end). The summation in Eq(22) is over all occupied states, $n$, and unoccupied (conduction) states, m. Also:

$$
F_{q m m}(k)=\left\langle n k\left|e^{-i q r}\right| m, k+q\right\rangle
$$


A direct evaluation of Eq(22) requires a complete quantum mechanical description of the crystal. For all but the simplest systems this is not possible, primarily because of computing limitations. For instance, within the self consistent field (SCF) Hartree-Fock (HF) scheme a reasonable description of the ground state obtains but excited states (virtual levels) may be in error by as much as 50\%. This is clearly a problem for Eq(22) where specific transitions (valence-conduction) are considered. One is left with two basic alternatives, both of which involve a "calibration" of sorts against data for $\epsilon(q=0, \omega)$ : a) in the semiempirical approach one may use a Hamiltonian with adjustable parameters or simply shift the energy gaps (this is known as "the scissors operator"), or b) use a simplified version of Eq(22) where the matrix elements, Eq(23), and band energies are taken as fitting parameters. This latter approach is used in the Oak Ridge code (Turner et al, 1982).

In a previous paper (zaider et al, 1990) we have described calculations of the dielectric response function for cubic ice; the results were obtained within the tight-binding approximation. We have evaluated directly $\mathrm{gq}(22)$, fitted available optical data $(q=0)$, and extended then the result in the $(w, q)$ plane. These results may be used directly in the Monte carlo transport code.

Our code is a modified version of our water-vapor based code, PROTON (zaider et al, 1983). Features common to both codes are not being further discussed here. This includes, for instance, the treatment of electron elastic scattering for which no data exist on condensed water other than at subexcitation energies.

The evaluation of $E(q, w)$ of $E q(22)$ requires computationallyintensive calculations of the single-particle band structure and 
wave functions. It is clear that direct evaluations of $\in(q, \omega)$ are possible only for a limited number of $(\omega, q)$ values; for other values analytic extrapolation and interpolation need to be used.

The problem of analytically continuing the energy loss function, $\operatorname{Im}[-1 / \epsilon(q, \omega)]$ at large $q$ values (at a fixed $\omega$ ) has been discussed by Dilion et al (1985) for the case of secondary electrons resulting from the ionization of molecules by charged particles. We have used an expression similar to that suggested in the cited paper, namely:

$$
\operatorname{Im}\left[-\frac{1}{\epsilon(q, \omega)}\right]=\sum_{i} \frac{A_{i}(\omega) q^{2 i}}{\left[\left(q^{2}-2 \times \omega\right)^{2}+8 I q^{2}\right]^{M}}
$$

I and $M$ are adjustable constants to be determined by fitting the low-q "data" (i.e. our direct calculations).

The single-collision (secondary electron) spectrum, $d \sigma(\omega, T) / d \omega$, for primary electrons with kinetic energy $T$ can be obtained by integrating the expressions, Eq(21), over all allowed momentum transfers. To extrapolate this spectrum at energies $\omega>100 \mathrm{eV}$ we have used the analytic form proposed by Green and sawada (1972):

$$
\frac{d \sigma_{G S}(\omega, T)}{d \omega}=\frac{A(T)}{[\omega-B(T)]^{2}+\Gamma^{2}(T)}
$$

where $\omega \in[0, T / 2]$ and $B$ and $\Gamma$ are $T$-dependent parameters. At energies $\omega>500 \mathrm{eV}$ the Mott-Moeller expression for the electron scattering cross section is used. Since the primary and secondary electrons are indistinguishable the spectra have to be corrected for exchange effects; this important correction has been made using the semi-empirical scheme suggested by Ritchie (1965).

2B. Nanodosimetric calculations for radon a particles The net result of transporting (in a Monte Carlo code) a charged 
particle event-by-event is a simulated track consisting of the geometrical positions of all energy deposition events as well as the amount of energy locally deposited at each interaction point. The calculation of a nanodosimetric (or microdosimetric) spectrum makes use, again, of the Monte Carlo technique. In an actual experiment the energy deposited in a fixed volume (the counter) by traversing particles is recorded. A calculation adopts the opposite procedure, namely, on one (or at most several) tracks a very large - i.e. sufficient to produce statistically significant results number of sampling volumes are placed and each time the total energy deposited is stored. The results below refer to a spherical volume: it is calculationally convenient and contradicts no biological evidence. The techniques described are, of course, valid for volumes of any shape.

The straightforward approach to produce a spectrum of specific energy is to "throw" random spheres in a volume (we shall call it generically a "box") completely enclosing the track. The efficiency of this procedure, measured as the ratio of successes (spheres containing at least one energy-transfer event) to the total number of spheres sampled, is generally less than one. "Missing" is in fact rather costly in terms of computing time since verifying the content of each sphere involves a loop over a large number of transfer points. It is therefore desirable to bring the efficiency as close to one as possible.

The volume around a track which has a sampling efficiency of exactly one is called the associated volume of that track (Lea, 1962 and Fig.4). Conceptually it can be built as follows: Centered at each transfer point place a sampling sphere. The union of all these spheres is the associated volume. It has the property 

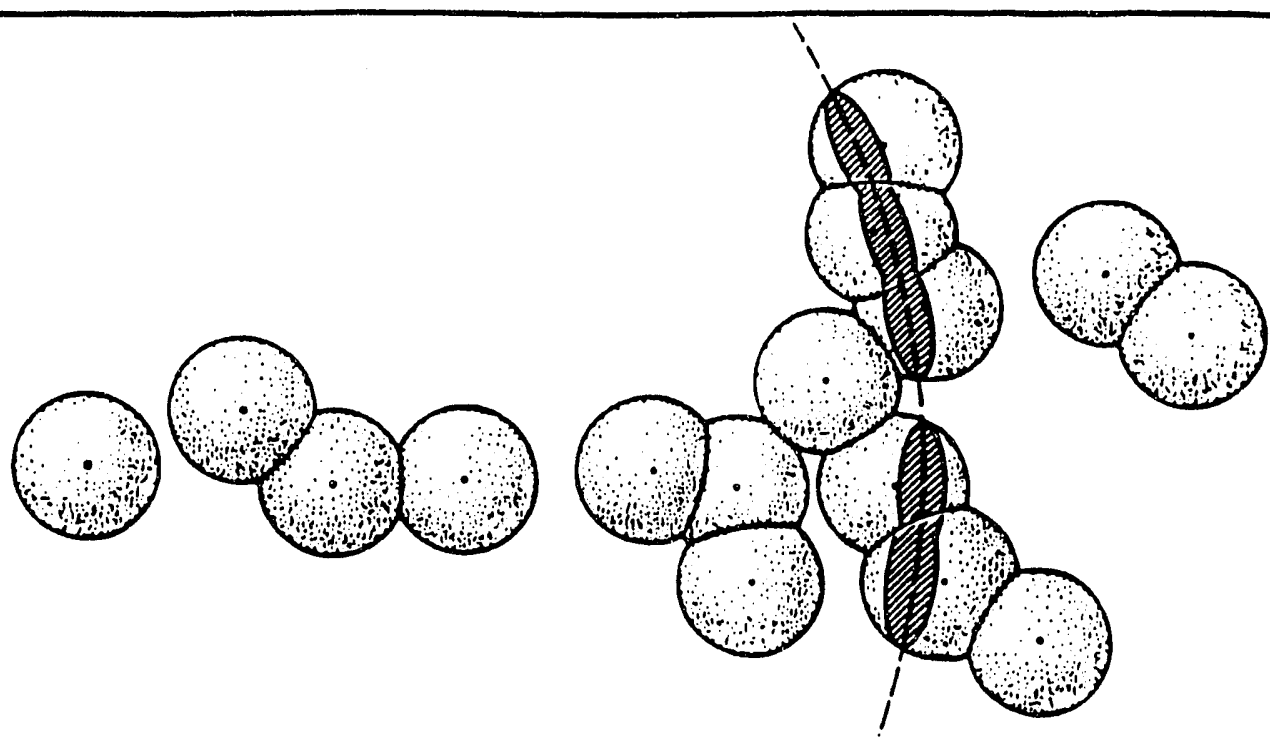

Figure 4 schematic description of the concepts of associated volume and associated surface (dashed area).

announced since, by its construction, any sphere placed with its center in it will be within at most one radius from at least one transfer point.

We have tevised (zaider and varma, 1992) a computational scheme for directly simulating the $A V$, that is for selecting spheres in the AV only, as follows: 1) select randomly a transfer point, 2) within a distance $R$ of this point place randomly a sphere of radius $R$, and 3 ) calculate the total energy in this sphere. The event thus obtained should be scored in the spectrum with a weight inversely proportional to the number of events in the sphere, $n$, since this method of selecting points in the $\mathrm{AV}$ is biased towards regions of high density of transfer points (straight sampling should be spatially uniform).

A key element in this method (and also in the definition of the AV) is the fact that the tracks intersecting the sphere originate uniformly from random points in space ano are isotropically distributed. This has been called $\mu$-randomness (Kellerer, 1980). In the calculation $\mu$-randomness translates in selecting spheres 
homogeneously.

Exposure by radon progeny of bronchial cells occurs under what might be called modified surface randomness (designated here by o-randomness): in its most basic configuration a cell is exposed to an isotropic distribution of a particles originating from a point at fixed distance, 1 , relative to the center of the cell (or site). Actual distributions are linear combinations of such elementary spectra. The unit-efficiency locus is now the intersection between the AV and a sphere of radius 1 centered at the origin of the track. We call this surface the associated surface (AS) of the track (see Fig.4). The simulation of the $A S$ is similar to that of the AV (the geometry of the problem is however more complicated). The main difference is in calculating the bias introduced by this method: the selection of spheres is a) proportional with the number of transfer points in the sphere which are within $[1-R, 1+R]$ from origin, and b) inversely proportional to the AS zone contained in the sphere.

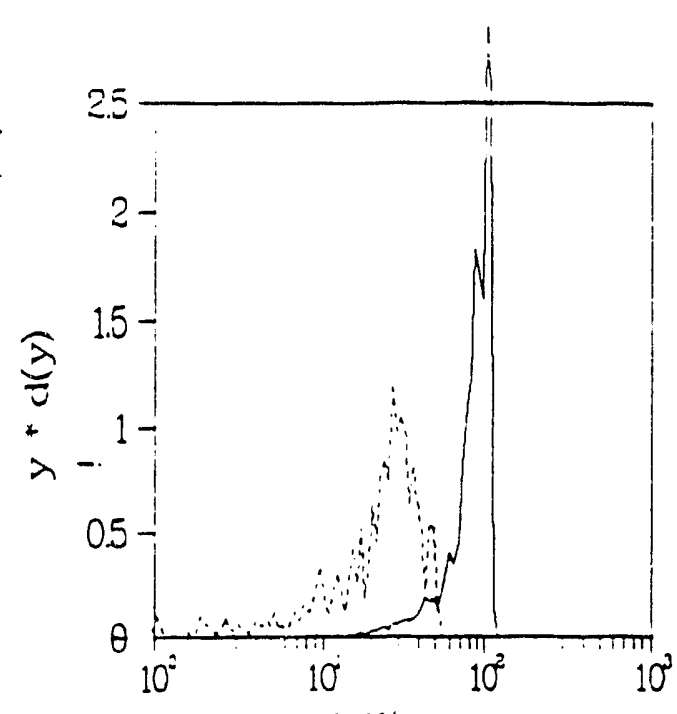

$\because \mathrm{kiv} / \mathrm{im}$

Figure 5 Monte Carlo calculation of microdosimetric spectra for spheres with $d=1 \mu \mathrm{m}$ and $\mathrm{d}=10 \mathrm{~nm}$. The $\alpha$ particle $(7 \mathrm{MeV})$ is at $3 \mu \mathrm{m}$ from the center. 
Monte Carlo nanodosimetric calculations have been performed for a variety of geometries. In Fig.5 we show two spectra: one for a sphere diameter $d=1 \mu \mathrm{m}$ (solid) and the other for $d=10 \mathrm{~nm}$. In both cases the 7-MeV $\alpha$ particle originated at a fixed distance $(3 \mu \mathrm{m})$ from the center of the sphere. The dramatic effect of the site diameter is evident. These calculations validate the application of the associated surface concept.

\section{DISCLAIMER}

This report was prepared as an account of work sponsored by an agency of the United States Government. Neither the United States Government nor any agency thereof, nor any of their employees, makes any warranty, express or implied, or assumes any legal liability or responsibility for the accuracy, completeness, or usefulness of any information, apparatus, product, or process disclosed, or represents that its use would not infringe privately owned rights. Reference herein to any specific commercial product, process, or service by trade name, trademark, manufacturer, or otherwise does not necessarily constitute or imply its endorsement, recommendation, or favoring by the United States Government or any agency thereof. The views and opinions of authors expressed herein do not necessarily state or reflect those of the United States Government or any agency thereof. 

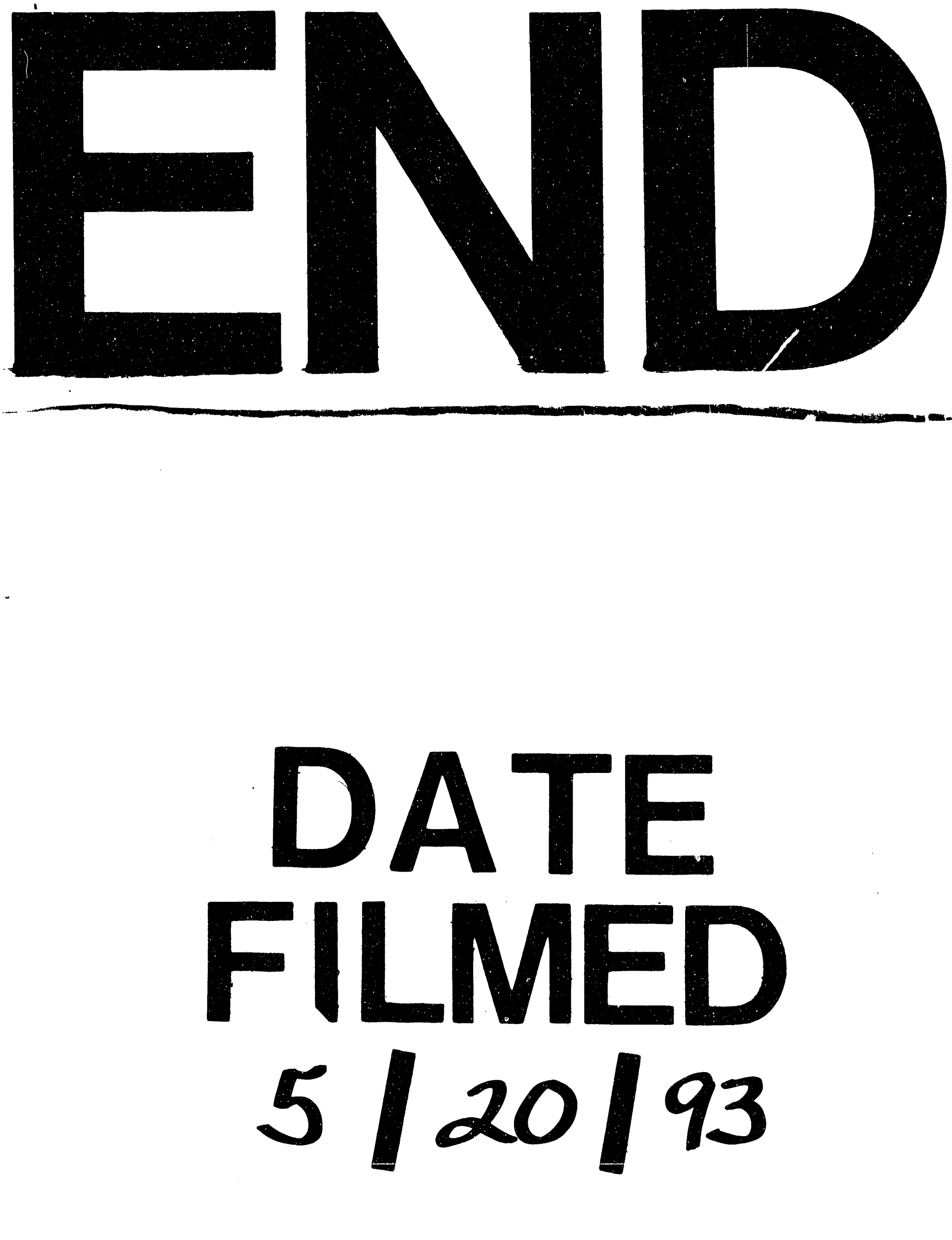
